\title{
ENFERMEDAD PERIODONTAL EN PACIENTES DIABÉTICOS DEL POLICLÍNICO DOCENTE "JULIÁN GRIMAU". SANTIAGO DE CUBA. CUBA
}

\author{
PERIODONTAL ILLNESS IN DIABETIC PATIENTS OF THE EDUCATIONAL POLICLINIC \\ "JULIÁN GRIMAU". SANTIAGO FROM CUBA. CUBA
}

Bárbara Olaydis Hechavarría Martínezªb, Josué Pujols Rues²c, Leonardo Núñez Antúnez 1ab

\section{RESUMEN}

Objetivo. Identificar el estado periodontal en pacientes con diabetes Mellitus. Material y método. Se efectuó un estudio descriptivo y transversal en 125 pacientes con Diabetes Mellitus del consultorio médico de la familia No. 33, pertenecientes al Policlínico Docente "Julián Grimau", en la ciudad de Santiago de Cuba, durante un año. Se utilizó el Índice Periodontal de Russell para determinar la severidad de la enfermedad periodontal y el Índice de análisis cuantitativo de Love para precisar el grado de higiene bucal. Resultados. La investigación mostró que la mayoría de los pacientes fueron del sexo femenino (92.5\%), prevaleciendo la periodontitis establecida para un (53.0\%). Conclusiones. En los pacientes diabéticos. La severidad de la enfermedad periodontal esta aumentada y la higiene bucal es deficiente. Se recomienda realizar estrategias de intervención educativa en los pacientes diabéticos para mejorar la higiene bucal.

Palabras clave: Diabetes mellitus; periodontitis; higiene bucal; enfermedad sistémica. (Fuente: DeCS).

\section{ABSTRACT}

Objetive: To identify the periodontal status of patients with Diabetes Mellitus. Methods: A descriptive and crosssectional study was conducted in 125 adult patients of the medical clinic from the family No. 33, belonging to the Educational Policlinic "Julian Grimau", in the city of Santiago from Cuba, during one year. The Periodontal Index of Russell was used to determine the severity of the periodontal illness and the Index of quantitative analysis of Love to specify the degree of buccal hygiene. Results: The present research showed that most of the patients were female $(92.5 \%)$, prevailing the established periodontitis for a $(53.0 \%)$. Conclusions: In diabetic patients there is an increment on the severity of periodontal disease and the oral hygiene was deficient. It is recommended to generate educational interventions in diabetic patients to improve their oral hygiene.

Key words: Diabetes mellitus; periodontitis; bucal hygiene; systemic disease. (Source: MeSH NLM).

${ }^{1}$ Facultad de Estomatología, Universidad de Ciencias Médicas, Santiago de Cuba, Cuba.

${ }^{2}$ Clínica Estomatológica "José Luis Tassende", Santiago de Cuba, Cuba.

a Especialista de 2do grado en Estomatología General Integral.

${ }^{b}$ MSc. Salud Bucal Comunitaria.

${ }^{c}$ Especialista de 1er grado en Estomatología General Integral.

\section{Correspondencia:}

Bárbara Olaydis Hechavarria Martínez.

Dirección: Facultad de Estomatología, Santiago de Cuba, Cuba.

Correo electrónico: barbara.hechavarria@medired.scu.sld.
Este es un artículo Open Access distribuido bajo la licencia Creative Commons Atribución-NoComercialCompartirlgual 4.0 


\section{INTRODUCCION}

La diabetes y la enfermedad periodontal también están presentes en niños y adolescentes. La gingivitis es más frecuente y severa en niños prepúber con diabetes, especialmente cuando el control metabólico es pobre. Entre los adolescentes, aproximadamente el $16 \%$ de los niños entre los 8 y 11 años con diabetes mellitus tiene periodontitis ${ }^{(1,2)}$.

Estudios tradicionales a finales de los años 60 demostraron que los jóvenes adultos insulinodependientes mostraron un incremento significativo en la destrucción de los tejidos periodontales comparado con personas sin diabetes $^{(3)}$.

Estos estudios mostraron, además, que individuos con diabetes del tipo 1 con cambios retínales experimentaron una mayor pérdida de soporte periodontal que aquellos sin esta complicación ocular ${ }^{(4,5)}$.

En Cuba, existen estudios publicados sobre las periodontopatías y su relación en la actualidad con diferentes enfermedades sistémicas y su etiología. Dichos estudios epidemiológicos plantean que más de $70 \%$ de la población adulta está afectada con enfermedades periodontales a partir de los 30 años, y más de un $15 \%$ se ha tratado con dicha patología, en muchos casos debido a enfermedades sistémicas como la Diabetes Mellitus ${ }^{(6,7)}$.

En Santiago de Cuba, varios autores han realizado investigaciones sobre las periodontopatías y su relación con la diabetes mellitus. A pesar de ello, en la actualidad sigue siendo un problema a resolver ya que son numerosos los pacientes que acuden a nuestros servicios con enfermedades periodontales en la atención primaria; sin embargo, nuestro sistema público dedica grandes recursos materiales y humanos en beneficio de la sociedad.

Teniendo en cuenta que en el Análisis de la Situación de Salud realizado en el servicio estomatológico perteneciente al Policlínico Docente "Julián Grimau" se pudo evidenciar que persiste un gran porcentaje de pacientes afectados por la enfermedad periodontal y gingival asociados con enfermedades endocrinas metabólicas como la Diabetes Mellitus y que, sin embargo, no existen estudios que caractericen esta problemática en el área, los autores se sintieron motivados a realizar el presente estudio para caracterizar a los pacientes diabéticos con enfermedad periodontal del consultorio médico de la familia $\mathrm{N}^{\circ}$. 33 del área de salud perteneciente al policlínico docente Julián Grimau.

\section{MATERIALES Y MÉTODO}

Se efectuó un estudio descriptivo y transversal en 125 pacientes con diabetes Mellitus del consultorio médico de la familia No. 33, pertenecientes al Policlínico Docente "Julián Grimau" en la ciudad de Santiago de Cuba, en el período comprendido de enero de 2015 a abril de 2016. En la fase de organización de la información se sostuvo un encuentro con el médico y la enfermera del consultorio de la familia $\mathrm{N}^{\circ} 33$ Petrocasa, para explicarles el objetivo del trabajo, establecer coordinaciones, participación y el apoyo en la obtención de la información.

A todos los pacientes encuestados se les pidió el consentimiento informado para participar de forma voluntaria en la investigación. Se les brindó previa información sobre ella, que no constituye ningún riesgo ni daño alguno para su salud y que debían de estar aptos mentalmente.

Para la recogida de las variables objeto de estudio, se les realizó un examen bucal minucioso al total de los pacientes diabéticos con enfermedad periodontal diagnosticados en un sillón dental con luz artificial. Nos auxiliamos de un espejo bucal, explorador y sonda periodontal milimetrada, además de la información ofrecida por el equipo del médico y enfermera de la familia del consultorio 33. Higiene bucal: se utilizó el índice de análisis cualitativo de Love que nos permitió evaluar el índice bucal en dos categorías: aceptable y deficiente.

Para la recolección de la información se confeccionó una planilla de recolección de datos que se llenó mediante la anamnesis y el examen físico intrabucal realizado en el servicio de estomatología del Policlínico Docente Julián Grimau de Santiago de Cuba y a través de la información obtenida por el médico y enfermera de la familia del consultorio 33 perteneciente a esta área de salud.

Una vez recogida la información se procesó en una computadora a través del sistema o programa estadístico SPSS versión 15,0. Se utilizaron como medidas de resumen la frecuencia absoluta y el porcentaje. La información obtenida se plasmó en tablas de dos entradas.

\section{RESULTADOS}

Los pacientes diabéticos con enfermedad periodontal según grupos de edades y sexo se aprecian en la tabla 1 , observando un predominio del sexo femenino con $66,4 \%$. El grupo de edad más afectado es el de 57 y más años de edad, representando el 53,6\%. Estos resultados pudieran estar relacionados con que la mayor cantidad de 
pacientes e ncontrados en el consultorio objeto de estudio, según dispensarización, correspondió al sexo femenino y mayores de 57 años, considerando además, que esta enfermedad aparecen con mayor frecuencia a medida que aumenta la edad.

Tabla 1. Pacientes diabéticos con enfermedad periodontal según grupos de edades y sexo

\begin{tabular}{lllllll}
\hline Grupos de edades & \multicolumn{3}{l}{ Femenino } & \multicolumn{2}{l}{ Masculino } & \multicolumn{2}{l}{ Total } \\
& No & $\%^{*}$ & No & $\%^{*}$ & No & $\%^{*}$ \\
\hline De 16 a 26 años & 2 & 1,6 & 1 & 0,8 & 3 & 2,4 \\
De 27 a 36 años & 3 & 2,4 & 1 & 0,8 & 4 & 3,2 \\
De 37 a 46 años & 5 & 4,0 & 3 & 2,4 & 8 & 6,4 \\
De 47 a 56 años & 27 & 21,6 & 16 & 12,8 & 43 & 34,4 \\
De 57 y más & 46 & 36,8 & 21 & 16,8 & 67 & 53,6 \\
\hline Total & 83 & 66,4 & 42 & 33,6 & 125 & 100 \\
\hline
\end{tabular}

*Porcentaje calculado en base al total de pacientes.

La tabla 2 refleja a los pacientes diabéticos con enfermedad periodontal según tipos de diabetes y severidad de la enfermedad periodontal. Se observa que del total de pacientes examinados (125), el 52\% pertenecen al tipo II de diabetes y un $48 \%$, al tipo I, prevaleciendo la periodontitis establecida con el $32,8 \%$, siendo la diabetes tipo II la más afectada por esta enfermedad, que alcanza el 16,8\%.

Tabla 2. Pacientes diabéticos según severidad de la enfermedad periodontal y tipos de diabetes

\begin{tabular}{llllllll}
\hline $\begin{array}{l}\text { Severidad de la enfermedad } \\
\text { periodontal }\end{array}$ & \multicolumn{9}{l}{ Tipos de Diabetes } \\
& $\begin{array}{l}\text { Tipo I } \\
\text { No }\end{array}$ & $\%^{*}$ & No & $\%^{*}$ & No & $\%^{*}$ \\
\hline Gingivitis & 12 & 9,6 & 11 & 8,8 & 23 & 18,4 \\
Gingivitis severa & 15 & 12 & 19 & 15,2 & 34 & 27,2 \\
Periodontitis establecida & 20 & 16 & 21 & $\mathbf{1 6 , 8}$ & 41 & $\mathbf{3 2 , 8}$ \\
Periodontitis terminal & 13 & 10,4 & 14 & 11,2 & 27 & 21,6 \\
\hline Total & 60 & $\mathbf{4 8}$ & 65 & $\mathbf{5 2}$ & 125 & 100 \\
\hline
\end{tabular}

${ }^{\star}$ Porcentaje calculado con base en el total de pacientes.

En la tabla 3 se constata que a un mayor tiempo de evolución de los pacientes con diabetes, hay una tendencia al aumento de severidad de la enfermedad periodontal. Así, se demuestra en los datos que en los pacientes diabéticos con tiempo de evolución de 0 a 5 años predominó la gingivitis severa (12\%), y de 6 a 10 años estuvo presente la periodontitis establecida $(12,8 \%)$, y en más de 11 años de evolución prevaleció la periodontitis terminal $(13,6 \%)$.

Tabla 3. Pacientes diabéticos según tiempo de evolución de la diabetes y severidad de la enfermedad periodontal

\begin{tabular}{lcccccc}
\hline Severidad de la enfermedad & \multicolumn{2}{c}{ Tiempo de evolución } \\
periodontal & \multicolumn{2}{c}{0 a 5 } & \multicolumn{2}{c}{6 a 10} & \multicolumn{2}{c}{11 y más } \\
& No & $\%^{*}$ & No & $\%^{*}$ & No & $\%^{*}$ \\
\hline Gingivitis & 10 & 8 & 10 & 8 & 3 & 2.4 \\
Gingivitis severa & 15 & $\mathbf{1 2}$ & 4 & 3,2 & 15 & 12 \\
Periodontitis establecida & 11 & 8,8 & 16 & $\mathbf{1 2 , 8}$ & 14 & 11,2 \\
Periodontitis terminal & 0 & 0 & 10 & 8 & 17 & $\mathbf{1 3 , 6}$ \\
\hline Total & 36 & 28,8 & 40 & 32 & 49 & 39,2 \\
\hline
\end{tabular}

${ }^{*}$ Porcentaje calculado con base en el total de pacientes. 
La relación del grado de severidad con el control metabólico en los pacientes diabéticos con enfermedad periodontal se expresa en la tabla 4. En los pacientes compensados predominó la gingivitis (13,6\%), mientras que en los descompensados la periodontitis establecida $(17,6 \%)$.

Tabla 4. Pacientes diabéticos según control metabólico de la diabetes y severidad de la enfermedad periodontal

\begin{tabular}{lccccc}
\hline $\begin{array}{l}\text { Severidad de la enfermedad } \\
\text { periodontal }\end{array}$ & \multicolumn{2}{c}{ Control metabólico } \\
& \multicolumn{3}{c}{ Compensados } & & \multicolumn{2}{c}{ Descompensados } \\
& Casos & No & $\%^{*}$ & No & $\%^{*}$ \\
Gingivitis & 23 & 17 & $\mathbf{1 3 , 6}$ & 6 & 4,8 \\
Gingivitis severa & 34 & 16 & 12,8 & 18 & 14,4 \\
Periodontitis establecida & 41 & 19 & 15,2 & 22 & $\mathbf{1 7 , 6}$ \\
Periodontitis terminal & 27 & 8 & 6,4 & 19 & 15,2 \\
\hline Total & 125 & 60 & 48 & 65 & 52 \\
\hline
\end{tabular}

*Porcentaje calculado con base en el total de pacientes.

La tabla 5 se identifica de los pacientes diabéticos según la higiene bucal, donde el mayor porcentaje se encuentra en los pacientes con una higiene bucal deficiente, con un $76,8 \%$. De igual forma, se pudo evidenciar que la periodontitis establecida es la más elevada en los pacientes con higiene bucal deficiente (24\%).

Tabla 5. Pacientes diabéticos según higiene bucal y severidad de la enfermedad periodontal

\begin{tabular}{lcccccc}
\hline \multicolumn{1}{c}{ Grado de severidad } & \multicolumn{2}{c}{ Higiene bucal } & \multicolumn{2}{c}{ Total } \\
& \multicolumn{2}{c}{ Aceptable } & \multicolumn{2}{c}{ Deficiente } & \multicolumn{2}{c}{} \\
& No & $\%^{*}$ & No & $\%^{*}$ & No & $\%$ \\
Gingivitis & 4 & 3,2 & 19 & 15,2 & 23 & 18,4 \\
Gingivitis severa & 12 & 9,6 & 22 & 17,6 & 34 & 27,2 \\
Periodontitis establecida & 11 & 8,8 & 30 & $\mathbf{2 4}$ & 41 & 32,8 \\
Periodontitis terminal & 2 & 1,6 & 25 & 20 & 27 & 21,6 \\
\hline Total & 29 & 23.2 & 96 & $\mathbf{7 6 , 8}$ & 125 & 100 \\
\hline
\end{tabular}

*Porcentaje calculado con base en el total de pacientes.

\section{DISCUSIÓN}

Los resultados obtenidos en la tabla 1 no coinciden con lo reflejado por González Gutiérrez ${ }^{(8)}$ y colaboradores quienes refieren que el sexo masculino fue el más afectado con la enfermedad periodontal. Coinciden respecto a la edad, ya que a medida que aumenta la misma, hay un crecimiento de periodontopatías, con un aumento de esta entre los 51 a 59 años, disminuyendo progresivamente según decrece la edad.

Olivencia ${ }^{(9)}$, en su estudio sobre diabetes y enfermedad periodontal refiere que esta enfermedad es un problema para muchas personas a medida que aumenta la edad. De hecho, la mayor parte de las personas de más de 40 años padecen de alguna forma de enfermedad periodontal, desde una leve inflamación de las encías hasta una infección periodontal severa.

Miranda y colaboradores, en un estudio sobre las consideraciones periodontales del paciente adulto mayor, señalan la presencia de periodontitis fulminante con destrucción acelerada de tejidos periodontales en los pacientes jóvenes y adultos con diabetes mellitus. Esta se caracteriza esta por pérdida de inserción periodontal rápida y progresiva con destrucción del hueso alveolar, presencia de micro abscesos gingivales múltiples y reacción gingival inflamatoria prolífera ${ }^{(10)}$.

Estudios científicos han demostrado que los jóvenes y adultos que padecen de diabetes tienen más probabilidad de desarrollar infecciones en las encías y enfermedad periodontal ${ }^{(11)}$. 
Esto les sucede especialmente a las personas con diabetes que no controlan bien el nivel de azúcar en su sangre. La diabetes también hace que la enfermedad periodontal sea más frecuente, más grave, y que se presente a una edad más temprana que en las personas que no tienen diabetes ${ }^{(12)}$.

Los datos de la tabla 2 coinciden con López Ramos $^{(13)}$,que demuestra que la severidad de la enfermedad periodontal pudiera estar relacionada con el tipo de diabetes, ya que hay un mayor número de pacientes con destrucción del tejido de soporte del diente en el tipo II a medida que avanza la enfermedad; sin embargo, hay una disminución de los pacientes en ambos tipos con periodontitis terminal.

En un estudio longitudinal de pacientes con diabetes Mellitus de tipo 2, los que padecían enfermedad periodontal avanzada presentaron un estado diabético significativamente peor que los que tenían enfermedad periodontal mínima ${ }^{(14)}$.

Otro estudio 4 demuestra que en personas con diabetes mellitus no dependientes de insulina, también llamada diabetes de tipo 2, la enfermedad periodontal es más severa y más prevalente que en personas sin diabetes.

Quisiguiña Guevara(7) resalta que el $81 \%$ de los pacientes diabéticos tipo II durante el examen bucodental fueron diagnosticados con enfermedad periodontal leve, moderada y severa, teniendo el mayor porcentaje la periodontitis leve con un $71 \%$.

Rodríguez Vázquez(12) es del criterio que el estado periodontal de los pacientes está relacionado con el tiempo de padecimiento de la diabetes mellitus, ya que pudo observar que a medida que avanza el tiempo de padecimiento de la diabetes mellitus, aumentaba la condición clínica de la enfermedad periodontal.

Un control metabólico inadecuado incrementó la severidad de la enfermedad periodontal y la necesidad de tratamientos más complejos acrecentando los recursos económicos necesarios para su resolución ${ }^{(15)}$.

La periodontitis severa se ha asociado con control metabólico y el seguimiento de estos pacientes, como mínimo dos años, sugiere que la periodontitis severa fue el factor de riesgo que comprometió su manejo. Podemos añadir que otros estudios han hallado una correlación de algunas de las complicaciones de la diabetes Mellitus, que incluyen retinopatía, nefropatía, neuropatía, proteinuria y complicaciones cardiovasculares con la severidad de la periodontitis ${ }^{(16)}$.
En las personas con diabetes Mellitus y mal control metabólico mantenido, se produce acumulación de los productos avanzados de glucosilación (PAG) no enzimática, lo que afecta la migración y la fagocitosis, tanto de los polimorfonucleares como de los mononucleares, lo que favorece el establecimiento de una flora subgingival que por maduración se irá transformando en flora predominantemente anaerobia gram negativa. Lo señalado anteriormente determina el aumento de la secreción de mediadores inflamatorios ya mencionados, los que mediarán en la destrucción del tejido conectivo, la reabsorción ósea y el desarrollo de una resistencia a la insulina en el tejido ${ }^{(17)}$.

La enfermedad periodontal severa puede tener malos efectos en el control de la diabetes porque hacen difícil y doloroso el masticar. Debido a estas molestias, la persona con diabetes puede decidir comer alimentos que son más fáciles de masticar, pero que pueden ser no apropiados para su plan de alimentación y que no garanticen un buen control metábolico ${ }^{(18)}$.

Los resultados de Rodríguez Vázquez no coinciden con los nuestros, al demostrar que los pacientes que tenían higiene bucal deficiente (81,2 \%) presentaron gingivitis severa, existiendo asociación significativa entre la deficiente higiene bucal y la severidad de la gingivitis, pues una mayor acumulación de placa dentobacteriana implica mayor frecuencia de pacientes con enfermedad periodontal. Se puede observar que los pacientes con eficiente higiene bucal presentaron condiciones menos graves de la enfermedad periodontal.

En otro estudio, los pacientes diabéticos tenían mala higiene bucal ya que el $56,6 \%$ de los pacientes presentaron gingivitis severa, mientras que el $12,8 \%$ tenía gingivitis leve, no coincidiendo con nuestro estudio ya que la gingivitis leve reveló un 18,4\% y la severa, un $27,2 \%$. Estos resultados corroboran los criterios ya establecidos sobre el importante rol de la higiene bucal en el inicio y desarrollo de los procesos inflamatorios crónicos que afectan los tejidos periodontales; es decir, a medida que empeora la higiene bucal, mayor es la respuesta inflamatoria que se producen en estos tejidos ${ }^{(19)}$.

La higiene bucal en los pacientes diabéticos es una piedra angular ya que la condición sistémica de base hace muy susceptibles a estos pacientes a manifestaciones inflamatorias en la encía, por lo que resultan vitales las actividades de educación para la salud para mantener la salud periodontal y lograr una adecuada higiene bucal $y$, de esta forma, atenuar las posibilidades de aparición de gingivitis y periodontitis. 
Se concluye que la mayoría de los pacientes diabéticos con enfermedad periodontal pertenecen al sexo femenino, siendo el grupo de edad más afectados los mayores de 57 años. Predominaron el tipo diabético II, el control metabólico descompensado y la higiene bucal deficiente con periodontitis establecida $y$, a mayor tiempo de evolución de la diabetes, prevaleció la periodontitis terminal.

La mejor forma de prevenir la periodontitis es mantener una correcta higiene oral para controlar los niveles de placa dental, pero en personas predispuestas, a pesar de una correcta higiene oral, puede tender a producirse la enfermedad. Por lo tanto, la higiene oral es un importante pilar de prevención de las enfermedades periodontales, pero no el único. Se debe acompañar de revisiones periódicas al especialista para que se pueda realizar un diagnóstico precoz de la enfermedad en caso de que se presente.

La ausencia de una adecuada higiene bucal es la principal causa de desarrollo de la enfermedad periodontal. Además, algunas personas, por causas genéticas, están más predispuestas que otras al desarrollo de esta patología. Mantener una buena higiene oral, cepillando los dientes de forma adecuada después de cada comida y usando la seda dental, es la mejor manera de prevenir el desarrollo de enfermedades de tipo periodontal y de mantener en buen estado la boca. Es también imprescindible acudir periódicamente al odontólogo, que no solo inspeccionará la dentadura, sino también el estado de las encías.

Contribuciones de autoría: $\mathrm{BOHM}$ participó en el diseño del estudio, redacción y análisis de resultados. JPR y LNA participaron en la revisión de la literatura, recolección de datos y procesamiento de datos. Todos los autores han participado en la revisión crítica del artículo y la aprobación de la versión final.

Fuente de financiamiento: autofinanciado.

Conflictos de interés: Los autores declaran no tener conflictos de interés en la publicación de este artículo.

\section{REFERENCIAS BIBLIOGRÁFICAS}

1. Smith P, Retamal I, Cáceres M, Romero A, Silva D, Arancibia R, Martínez C. Diabetes y su impacto en el territorio periodontal. Rev. Clin. Periodoncia Implantol. Rehabil Oral [Internet]. 2012 [citado 23 de Octubre de 2015]; 5(2). Disponible en: http://www.scielo.cl/scielo.php?pid=s071901072012000200008\&script=sci_arttext.
2. Luna M. Diabetes y enfermedad periodontal: 10 preguntas con respuesta. [Internet] Madrid. 2014 [citado 23 de Octubre de 2015] Disponible en: http://www.abc.es/salud/noticias/20141113/abcidiabetes-salud-oral-201411131012.html.

3. Coke. Enfermedad periodontal en paciente diabético. [Internet] Chile. [Artículo en línea] 2012 [citado 23 de Octubre de 2015] Disponible en: http://html.rincondelvago.com/enfermedadperiodontal-en-paciente-diabetico.html.

4. Díaz Romero RM, F, Álvarez Fuente FV. Diabetes e infección periodontal. [Internet], [Artículo en línea] 2015 [citado 5 de Diciembre de 2015] Disponible en: http://www.intramed.net/contenidover.asp?contenidol $\mathrm{D}=72517$.

5. Complicaciones de la diabetes: Enfermedad periodontal. Blog Diabetes [Internet]. 2014 [citado 23 de Octubre de 2015] Disponible en: http://blog.diabetes.ascensia.es/blog/index.php/compli caciones-de-la-diabetes-enfermedad-periodontal

6. Blair L. La estrecha relación entre la enfermedad periodontal y la diabetes. Blog de la salud. [Internet]. 2016 [citado 7 de Abril de 2016] Disponible en: https://www.elblogdelasalud.info/la-estrecha-relacionentre-la-enfermedad-periodontal-y-la-diabetes/10387

7. Quisiguiña Guevara N. Relación de la enfermedad periodontal en pacientes diabéticos tipo 2, del club de diabéticos en el Hospital IESS Riobamba a partir de diciembre/2011, hasta agosto/2012.[Internet], [Artículo en línea] 2012 [citado 5 de Diciembre de 2015] Disponible en: <http://www.dspace.uce.edu.ec/handle/25000/524

8. González Gutiérrez A, Trasancos Delgado $M$, González Cordero AE, Casanova Moreno MC. Comportamiento de la enfermedad periodontal en pacientes diabéticos. Policlínico «Pedro Borrás Astorga. Rev Ciencias Méd Pinar del Río [Internet]. 2012 [citado 23 de Octubre de 2015 ]; 16(1). Disponible

en:http://scielo.sld.cu/scielo.php?script=sci_arttext\&pi $\mathrm{d}=$ S1561-31942012000100018

9. Olivencia P. Diabetes y enfermedad periodontal: Blog cuida tu boca. [Internet]. 2016 [citado 10 de Abril de 2016] Disponible en: http://www.vitis.es/comoprevenir-la-enfermedad-periodontal-para-ayudar-acontrolar-la-diabetes/

10. Miranda Galvis M, Montoya Zuluaga YP, Saldarriaga Saldarriaga A. Diabetes y enfermedad periodontal: hacia un modelo clínico bidireccional. Rev. Nac. de Odontol. [Internet]. 2012 [citado 23 de Oct de 2015]; 8(14). Disponible en: http://revistas.ucc.edu.co/index.php/od/article/viewFile /285/296

11. Cómo prevenir la enfermedad periodontal para ayudar a controlar la diabetes. Blog cuida tu boca. [Internet]. 2016 [citado 10 de Abr de 2016]. Disponible en: http://www.cuidatuboca.com/diabetes-enfermedadperiodontal/

12. Rodríguez Vázquez $\mathrm{Y}$, Alemán Hernández $\mathrm{E}$, Ricardo Rodríguez Llanes R, Valdivia Cano MI, Galá Piloto E, Díaz Apesteguía G. Enfermedad periodontal inmunoinflamatoria crónica en pacientes diabéticos en edad pediátrica. Rev Cub de Estomatol. [Internet]. 2015 [citado 23 de Octubre de 2015]; 52. Disponible en: 
13. López Ramos R. Cómo se desarrolla la enfermedad periodontal.[Internet]. 2012. [Artículo en línea] .[citado 23 de Octubre de 2015]. Disponible en: http://www.scielo.cl/scielo.php?pid=s0719010720120 00200008\&script $=$ sci_arttext $14-$ Marble M, Alvarez BJ. Oral health also affected by disease. [Internet]. 2012 [citado 23 de Octubre de 2015]. Disponible en: http://web12.epenet.com./

14. Henderson CW. Estrogen, osteoporosis, periodontal disease and tooth loss. Women's Health Weekly [Internet]. 2012 [citado 23 de Octubre de 2015]. Disponible en: http://web12.epenet.com./

15. Consideraciones Periodontales del Paciente Adulto Mayor. [Internet]. 2015. [Artículo en línea], [citado 23 de Octubre de 2015]. Disponible en: http://go.microsoft.com/fwlink/?Linkld=121315

16. Guillen D. Diabetes en pacientes con enfermedad periodontal.[Internet]. 2015. [Artículo en línea], [citado 23 de Octubre de 2015]. Disponible en: https://prezi.com/eoq8n8qdp9bz/diabetes-enpacientes-con-enfermedad-periodontal

17. Ramírez Bonilla J. Gingivitis asociada a la diabetes. [Internet]. 2012. [Artículo en línea], [citado 23 de Octubre de 2015]. Disponible en:
18. Pretel-Tinoco C, Chávez Reátegui B. Enfermedad periodontal como factor de riesgo de condiciones sistêmicas. Rev. Estomatol Herediana. [Internet]. 2013. [citado 23 de Octubre de 2015]; 23(4). Disponible en: http://www.upch.edu.pe/vrinve/dugic/revistas/index.ph $\mathrm{p} / \mathrm{REH} /$ article/viewFile/18/11

19. Juárez Rolando $P$, Chahin Julio R, Vizcaya Maytena M, Arduña Emma I. Evaluación clínica de pacientes diabéticos relacionada al estado de salud bucal. [Internet]. 2012. [Artículo en línea]. [citado 23 de Octubre de 2015].Disponible en: http://odn.unne.edu.ar/12.pdf 